RIMS-851

December 1991

\title{
Partition Functions and Topology-Changing Amplitudes in the 3D Lattice Gravity of Ponzano and Regge
}

\author{
HiROSI OOGURI \\ Research Institute for Mathematical Sciences \\ Kyoto University, Kyoto 606, Japan
}

\begin{abstract}
We define a physical Hilbert space for the three-dimensional lattice gravity of Ponzano and Regge and establish its isomorphism to the one in the $I S O(3)$ Chern-Simons theory. It is shown that, for a handlebody of any genus, a Hartle-Hawking-type wave-function of the lattice gravity transforms into the corresponding state in the Chern-Simons theory under this isomorphism. Using the Heegaard splitting of a three-dimensional manifold, a partition functions of each of these theories is expressed as an inner product of such wavefunctions. Since the isomorphism preserves the inner products, the partition functions of the two theories are the same for any closed orientable manifold. We also discuss on a class of topology-changing amplitudes in the lattice gravity and their relation to the ones in the Chern-Simons theory.
\end{abstract}

\footnotetext{
^ e-mail addresses : ooguri@jpnrifp.bitnet and ooguri@kekvax.kek.ac.jp
} 
In 1968, Ponzano and Regge derived the following asymptotic form of the RacahWigner $6 j$-symbol for large angular momenta $j_{i}$ 's $[1]$.

$$
\begin{gathered}
(-1)^{\sum_{i=1}^{6} j_{i}}\left\{\begin{array}{ccc}
j_{1} & j_{2} & j_{3} \\
j_{4} & j_{5} & j_{6}
\end{array}\right\} \sim \\
\sim \frac{1}{\sqrt{12 \pi V}} \cos \left(S_{\text {Regge }}+\pi / 4\right) \\
\left(j_{i} \in \mathbf{Z}_{\geq 0}\right) .
\end{gathered}
$$

To explain the notations in the right hand side, it is useful to imagine a tetrahedron and associate $j_{i}$ 's to its edges as in Fig. 1. In the following, we call this as coloring of the tetrahedron. Since the $6 j$-symbol has the tetrahedral symmetry, we can uniquely associate it to the colored tetrahedron. Now we regard $\left(j_{i}+\frac{1}{2}\right)$ as a length of the $i$-th edge of the tetrahedron. The factor $V$ in the right hand side of (1) is defined as a volume of such a tetrahedron, and $S_{\text {Regge }}$ is given by

$$
S_{\text {Regge }}=\sum_{i=1}^{6} \theta_{i}\left(j_{i}+\frac{1}{2}\right)
$$

where $\theta_{i}$ is the angle between the outward normals of the two faces separated by the $i$-th edge.

What is remarkable about this formula is that $S_{\text {Regge }}$ is nothing but the Regge action[2] for the single tetrahedron. Suppose there is a three-dimensional manifold $M$ which is decomposed into a collection of tetrahedra. If we assume that each tetrahedron is filled in with flat space and the curvature of $M$ is concentrated on the edges of the tetrahedron, a metric $g_{\mu \nu}$ on $M$ is specified once the length $\left(j+\frac{1}{2}\right)$ of each edge is fixed. The EinsteinHilbert action $\int d^{3} x \sqrt{g} R$ is then a function of $j$ 's on the edges and it is given by summing the Regge action (2) over all the tetrahedra in $M$. Thus, as a model for the three-dimensioal Einstein gravity, Ponzano and Regge considered a lattice statistical model whose dynamical variables are the angular momenta $j$ 's on the edges and whose weight is given by a product of the $6 j$-symbols over all the tetrahedra in $M$ (including the sign-factor $(-1)^{\sum_{i} j_{i}}$ in the left hand side of $(1))$.

In the lattice gravity, we sum over geometries of $M$ based on its simplicial decomposition. In one approach, size and shape of each simplex are fixed, and the quantum 
flctuation of the geometry is evaluated by summing over all the possible ways of gluing the simplices together. The recent studies on two-dimensional gravity are mostly based on this approach[3]. In the other approach, one fixes the lattice structure and sums over the lattice lengths [2]. The lattice model of Ponzano and Regge belongs to the latter approach.

In both of these approaches, it is important to know if the lattice model has a nice continuum limit. In this respect, it has already been pointed out by Ponzano and Regge that their lattice model can be made scale-invariant with appropriate modification of the statistical weight. Let us take the tetrahedron in Fig. 1, and decompose it into four small tetrahedra as in Fig. 2. There are four edges inside of the original tetrahedron, and we put angular momenta $l_{1}, \ldots, l_{4}$ on them. Corresponding to the four tetrahedra, we consider the following product of the $6 j$-symbols.

$$
(-1)^{\sum_{i} l_{i}}\left\{\begin{array}{lll}
j_{1} & j_{2} & j_{3} \\
l_{1} & l_{2} & l_{3}
\end{array}\right\}\left\{\begin{array}{lll}
j_{4} & j_{6} & j_{2} \\
l_{3} & l_{1} & l_{4}
\end{array}\right\}\left\{\begin{array}{lll}
j_{3} & j_{4} & j_{5} \\
l_{4} & l_{2} & l_{1}
\end{array}\right\}\left\{\begin{array}{lll}
j_{1} & j_{5} & j_{6} \\
l_{4} & l_{3} & l_{2}
\end{array}\right\}
$$

Now we are going to sum this weight over the coloring $l_{i}$ on the internal edges. The summation can be performed analytically if we multiply an additional factor $\prod_{i=1}^{4}\left(2 l_{i}+1\right)$ to the summand (3). By using the identity due to Biedenharn and Elliot, the summation over $l_{1}$ can be done as

$$
\begin{aligned}
& \sum_{l_{1}}\left(2 l_{1}+1\right)(-1)^{\sum_{i} l_{i}}\left\{\begin{array}{lll}
j_{4} & j_{6} & j_{2} \\
l_{3} & l_{1} & l_{4}
\end{array}\right\}\left\{\begin{array}{lll}
j_{3} & j_{4} & j_{5} \\
l_{4} & l_{2} & l_{1}
\end{array}\right\}\left\{\begin{array}{lll}
j_{1} & j_{5} & j_{6} \\
l_{4} & l_{3} & l_{2}
\end{array}\right\} \\
& =(-1)^{\sum_{i} j_{i}}\left\{\begin{array}{lll}
j_{1} & j_{2} & j_{3} \\
j_{4} & j_{5} & j_{6}
\end{array}\right\}\left\{\begin{array}{lll}
j_{1} & j_{5} & j_{6} \\
l_{4} & l_{3} & l_{2}
\end{array}\right\} \text {. }
\end{aligned}
$$

We can then sum over $l_{4}$ using the orthonormality of the $6 j$-symbol

$$
\sum_{l_{4}}\left(2 l_{4}+1\right)\left\{\begin{array}{lll}
j_{1} & j_{5} & j_{6} \\
l_{4} & l_{3} & l_{2}
\end{array}\right\}^{2}=\frac{1}{2 j_{1}+1}
$$


Thus we are left with the sum over $l_{2}$ and $l_{3}$ as

$$
\begin{aligned}
& \sum_{l_{1}, \ldots, l_{4}}(-1)^{\sum_{i} l_{i}}\left(2 l_{1}+1\right)\left(2 l_{2}+1\right)\left(2 l_{3}+1\right)\left(2 l_{4}+1\right) \\
& \quad \times\left\{\begin{array}{lll}
j_{1} & j_{2} & j_{3} \\
l_{1} & l_{2} & l_{3}
\end{array}\right\}\left\{\begin{array}{lll}
j_{4} & j_{6} & j_{2} \\
l_{3} & l_{1} & l_{4}
\end{array}\right\}\left\{\begin{array}{lll}
j_{3} & j_{4} & j_{5} \\
l_{4} & l_{2} & l_{1}
\end{array}\right\}\left\{\begin{array}{lll}
j_{1} & j_{5} & j_{6} \\
l_{4} & l_{3} & l_{2}
\end{array}\right\} \\
& =(-1)^{\sum_{i} j_{i}}\left\{\begin{array}{lll}
j_{1} & j_{2} & j_{3} \\
j_{4} & j_{5} & j_{6}
\end{array}\right\} \quad \frac{1}{2 j_{1}+1} \sum_{\left|l_{2}-l_{3}\right| \leq j_{1} \leq l_{2}+l_{3}}\left(2 l_{2}+1\right)\left(2 l_{3}+1\right) .
\end{aligned}
$$

However, the sum over $l_{2}$ and $l_{3}$ in the right hand side is divergent. In order to regularize it, we cut off the summation by $l_{i} \leq L$ and rescale the summand of (4) by multiplying a factor $\Lambda(L)^{-1}$, where

$$
\Lambda(L)=\frac{1}{2 j_{1}+1} \sum_{\substack{l_{2}, l_{3} \leq L \\\left|l_{2}-l_{3}\right| \leq j_{1} \leq l_{2}+l_{3}}}\left(2 l_{2}+1\right)\left(2 l_{3}+1\right)
$$

For a sufficiently large value of $L, \Lambda(L)$ becomes independ on $l_{1}$ and behaves as $\Lambda(L) \sim$ $4 L^{3} / 3$ for $L \rightarrow \infty$. After multiplying this factor, we can take $L$ to $\infty$ and the divergence is removed. Thus, with the additional factor $\Lambda(L)^{-1} \prod_{i}\left(2 l_{i}+1\right)$, the sum of $(3)$ over the coloring $l_{i}$ on the internal edges in Fig. 2 reproduces the weight

$$
(-1)^{\sum_{i} j_{i}}\left\{\begin{array}{lll}
j_{1} & j_{2} & j_{3} \\
j_{4} & j_{5} & j_{6}
\end{array}\right\}
$$

for the original tetrahedron in Fig. 1.

Based on this observation, Ponzano and Regge defined a partition function $Z_{M}$ for the manifold $M$ by

$$
Z_{M}=\lim _{L \rightarrow \infty} \sum_{\{j: j \leq L\}} \prod_{\text {vertices }} \Lambda(L)^{-1} \prod_{\text {edges }}(2 j+1) \prod_{\text {tetrahedra }}(-1)^{\sum_{i} j_{i}}\left\{\begin{array}{ccc}
j_{1} & j_{2} & j_{3} \\
j_{4} & j_{5} & j_{6}
\end{array}\right\}
$$

Due to the identity (4), $Z_{M}$ is invariant under the refinement of any tetrahedron in $M$ into four smaller tetrahedra. Namely this lattice model is at a fixed point the renormalization group transformation ${ }^{\star}$.

* Although the divergence due to the scale-invariance of the model is regularized in (6) by multiplying the factor $\Lambda(L)^{-1}$, it is not obvious that $Z_{M}$ defined in the above is finite. We will examine this point in Section 3. 
Because of this property, one may suspect that the lattice gravity of Ponzano and Regge can be related to some quantum field theory in the continuum. Although there have been some works on physical interpretation of this model[4], little progress had been made on the continuum limit of this model until recently. Last year, Turaev and Virostudied the $q$-analogue of the Ponzano-Regge model, and found that its partition function is invariant under a class of transformations larger than the renormalization group in the above. Moreover they have shown that any two tetrahedral decompositions of $M$ can be related by a sequence of such transformations. Therefore the partition is independent of the tetrahedral decomposition and depends only on the topology of $M$. Although they have studied the $q$ analogue, their argument is directly applicable to the original model of Ponzano and Regge. Thus it is natural to expect that the model of Ponzano and Regge and its $q$-analogue by Turaev and Viro are equivalent to some topological field theories. Indeed, in the paper [5], Turaev and Viro have conjectured that the partition function of their $q$-analogue model is equal to the absolute value square of the partition function of the $S U(2)$ Chern-Simons theory[6] of level $k\left(q=e^{2 \pi i /(k+2)}\right)$ when the manifold $M$ is orientable.

In the previous paper[7], the author and Sasakura have examined physical states in the lattice gravity of Ponzano and Regge and suggested that they are related to physical states of the $I S O(3)$ Chern-Simons theory whose action is given by

$$
S_{C S}(e, \omega)=\int d^{3} x e^{a} \wedge\left(d \omega^{a}+\epsilon^{a b c} \omega^{b} \wedge \omega^{c}\right),
$$

where $e^{a}$ and $\omega^{a}(a=1,2,3)$ are one-forms on $M$ with adjoint indices of $S O(3)$. If we identify them as a dreibein and a spin-connection following the observation by Witten, the action $S_{C S}$ may be regarded as the Einstein-Hilbert action $\int e \wedge R$ in the first order formalism.

In this paper, we extend the analysis of [7] and show that the partition function of the lattice gravity of Ponzano and Regge agrees with the one of the $I S O(3)$ Chern-Simons theory for any orientable manifold. This result corresponds to the $k \rightarrow \infty$ limit of the cojecture by Turaev and Viro. In Section 2, we define a physical Hilbert space for the lattice gravity and establish its isomorphism to the physical Hilbert space of the Chern-Simons theory. We show later in Section 4 that this isomorphism preserves the inner products of the two Hilbert spaces. In Section 3, we compute the Hartle-Hawking-type wave-functions 
of the lattice gravity for a handlebody of any genus and show that it transformes into the corresponding state in the Chern-Simons theory under the isomorphism. By gluing Hartle-Hawking-type wave-functions, one can compute a partition function for any closed orientable manifold. We check in Section 4 that this gluing procedure is compatible with the isomorphism. Therefore the partition functions of these two theories are the same, as far as they are finite. We also study some class of topology-changing amplitudes in the lattice gravity and their relation to the ones in the Chern-Simons theory. In the last section, we discuss on interpretations of these results and their extensions.

In the course of this work, the author was informed of a paper by Turaev[9] where he announces to have proven the equivalence of the $q$-analogue lattice model and the ChernSimons theory for finite $k$. Details of his derivation not being available, it is not clear to the author how his approach is related to the one presented here.

\section{Wave-Functions}

In the lattice gravity of Ponzano and Regge, one can define a discretized verion of the Wheeler-DeWitt equation which characterizes physical states in the theory. On the other hand, in the $I S O(3)$ Chern-Simons theory, a physical state is given by a gauge-invariant half-density $\Phi(\omega)$ on the moduli space of a flat $S O(3)$ connection $\omega$ on a two-dimensional surface $\Sigma$. In this section, we establish a correspondence between physical states in the lattice gravity and in the continuum Chern-Simons theory. This correspondence will be used in the later sections to compare partition functions and topology-changing amplitudes in those two theories.

First we should clarify what we mean by physical states in the lattice gravity. To motivate our definition of physical states, let us consider a closed three-dimensional manifold $M$ and decompose it into three parts, $M_{1}, M_{2}$ and $N$, as in Fig. 3, where $N$ has a topology of $\Sigma \times[0,1]$ with $\Sigma$ being a closed orientable two-dimensional surface, and $M_{i}(i=1,2)$ has a boundary which is isomorphic to $\Sigma$. The manifold $M$ is reconstructed by gluing the boundaries of $N$ with $\partial M_{1}$ and $\partial M_{2}$.

Corresponding to this decomposition of $M$, the partition function $Z_{M}$ of the manifold $M$ can be expressed as a sum of products of three components each of which is associated to $M_{1}, M_{2}$ and $N$. To find such an expression, we note that the partition function $Z_{M}$ 
is independent of a choice of tetrahedral decomposition of $M$. Therefore we can place tetrahedra in $M$ in such a way that $M_{1}, M_{2}$ and $N$ do not share a tetrahedron, namely their boundaries are triangulated by the faces of the tetrahedra. Corresponding to this tetrahedral decomposition, we can express $Z_{M}$ as

$$
Z_{M}=\sum_{\substack{c_{1} \in C\left(\Delta_{1}\right) \\ c_{2} \in C\left(\Delta_{2}\right)}} Z_{M_{1}, \Delta_{1}}\left(c_{1}\right) \Lambda^{-n\left(\Delta_{1}\right)} P_{\Delta_{1}, \Delta_{2}}\left(c_{1}, c_{2}\right) \Lambda^{-n\left(\Delta_{2}\right)} Z_{M_{2}, \Delta_{2}}\left(c_{2}\right)
$$

Here $\Delta_{i}(i=1,2)$ denotes the triangulations of the boundary $\partial M_{i}, C\left(\Delta_{i}\right)$ is a set of all the possible colorings on $\Delta_{i}$, and $n\left(\Delta_{i}\right)$ is a number of vertices on $\Delta_{i}{ }^{\star}$. The factor $Z_{M_{i}, \Delta_{i}}\left(c_{i}\right)$ is given by the sum over all the possible coloring on the edges interior of $M_{i}$

$$
\begin{aligned}
Z_{M_{i}, \Delta}\left(c_{i}\right)= & \prod_{\text {edges on } \Delta_{i}}(-1)^{2 j} \sqrt{2 j+1} \\
& \times \sum_{\substack{\text { coloring } \\
\text { interior of } M_{i}}} \prod_{\begin{array}{c}
\text { vertices } \\
\text { interior of } M_{i}
\end{array}}(2 j+1) \\
& \times \prod_{\text {tetrahedra in } M_{i}}(-1)^{\sum_{i} j_{i}}\left\{\begin{array}{lll}
j_{1} & j_{2} & j_{3} \\
j_{4} & j_{5} & j_{6}
\end{array}\right\},
\end{aligned}
$$

where we keep fixed the coloring $c_{i}$ on the edges on $\partial M_{i}$ (Fig. 4). Similarly $P_{\Delta_{1}, \Delta_{2}}\left(c_{1}, c_{2}\right)$ is given by a sum over all the possible colorings on the interior edges of $N$ with fixed colorings $c_{1}$ and $c_{2}$ on $\partial N \simeq \Sigma+\Sigma$.

Since $P_{\Delta_{1}, \Delta_{2}}$ is independent of the tetrahedral decomposition of the interior of $N$, it satisfies the following remarkable property,

$$
\sum_{c_{2} \in C\left(\Delta_{2}\right)} P_{\Delta_{1}, \Delta_{2}}\left(c_{1}, c_{2}\right) \Lambda^{-n\left(\Delta_{2}\right)} P_{\Delta_{2}, \Delta_{3}}\left(c_{2}, c_{3}\right)=P_{\Delta_{1}, \Delta_{3}}\left(c_{1}, c_{3}\right) .
$$

Therefore we can define an operator $\mathcal{P}$

$$
\mathcal{P}\left[\phi_{\Delta}\right](c)=\sum_{c^{\prime} \in C(\Delta)} P_{\Delta, \Delta}\left(c, c^{\prime}\right) \Lambda^{-n(\Delta)} \phi_{\Delta}\left(c^{\prime}\right),
$$

which acts as a projection operator $(\mathcal{P} \cdot \mathcal{P}=\mathcal{P})$ on a space of functions on $C(\Delta)$. By using

* For conciseness of equations, here and in the following, we do not write the cut-off parameter $L$ explicitly. 
(9), we can rewrite (8) as

$$
Z_{M}=\sum_{c_{1}, c_{2}} \mathcal{P}\left[Z_{M_{1}, \Delta_{1}}\right]\left(c_{1}^{\prime}\right) \Lambda^{-n\left(\Delta_{1}\right)} P_{\Delta_{1}, \Delta_{2}}\left(c_{1}, c_{2}\right) \Lambda^{-n\left(\Delta_{2}\right)} \mathcal{P}\left[Z_{M_{2}, \Delta_{2}}\right]\left(c_{2}^{\prime}\right)
$$

One sees that "states" propagating from $M_{1}$ to $M_{2}$ through $N$ are projected out by $\mathcal{P}$. Thus it is natural to define a physical Hilbert space $H(\Delta)$ for the triangulated surface $\Sigma$ as a subspace projected out by $\mathcal{P}$, i.e.

$$
\phi_{\Delta}(c) \in H(\Delta) \quad \Longleftrightarrow \quad \phi_{\Delta}=\mathcal{P}\left[\phi_{\Delta}\right]
$$

Since $P_{\Delta, \Delta}$ is associated to the topology $\Sigma \times[0,1]$, we may regard it as a time evolution operator in the lattice gravity. Therefore it should be appropriate to call the physical state condition (10) as a discretized version of the Wheeler-DeWitt equation. We define an inner product in $H(\Delta)$ by

$$
\left(\phi_{\Delta}, \phi_{\Delta}^{\prime}\right)=\sum_{c, c^{\prime} \in C(\Delta)} \phi_{\Delta}(c) \Lambda^{-n(\Delta)} P_{\Delta, \Delta}\left(c, c^{\prime}\right) \Lambda^{-n(\Delta)} \phi_{\Delta}^{\prime}\left(c^{\prime}\right) .
$$

It is easy to see that $Z_{M_{1}, \Delta}(c)$ and $Z_{M_{2}, \Delta}(c)$ are real solutions to the Wheeler-DeWitt equation (10) and the partition function $Z_{M}$ is given by their inner product

$$
Z_{M}=\left(Z_{M_{1}, \Delta}, Z_{M_{2}, \Delta}\right)
$$

Although this definition of $H(\Delta)$ depends of the triangulation $\Delta$ of $\Sigma$, there is a natural isomorphism given by the map $P_{\Delta_{1}, \Delta_{2}}$ between $H\left(\Delta_{1}\right)$ and $H\left(\Delta_{2}\right)$ for any two triangulations $\Delta_{1}, \Delta_{2}$. Due to the equation (9), the map $P_{\Delta_{1}, \Delta_{2}}$ preserves the inner product defined by (11). It also follows from (9) that the map $P_{\Delta_{1}, \Delta_{2}}$ has an inverse and it is given by $P_{\Delta_{2}, \Delta_{1}}$. Thus we may choose an arbitrary triangulation in defining the physical Hilbert space for $\Sigma$.

On the other hand, the physical Hilbert space $H_{C S}$ of the $I S O(3)$ Chern-Simons theory consists of half-densities on the moduli space of a flat $S O(3)$ connection on $\Sigma$. To see this, 
we consider the topology $N=\Sigma \times[0,1]$ again, and decompose the dreibein $e^{a}$ and the spin-connection $\omega^{a}(a=1,2,3)$ as

$$
\begin{gathered}
e^{a}=\sum_{i=1,2} e_{i}^{a} d x^{i}+e_{0}^{a} d t, \quad \omega^{a}=\sum_{i=1,2} \omega_{i}^{a} d x^{i}+\omega_{0}^{a} d t \\
\left(x^{1}, x^{2}\right) \in \Sigma, \quad t \in[0,1] .
\end{gathered}
$$

Corresponding to this decomposition, the Chern-Simons action (7) takes the form,

$$
S_{C S}(e, \omega)=\int d t d^{2} x \epsilon^{i j}\left(e_{j}^{a} \partial_{t} \omega_{i}^{a}+e_{0}^{a} F_{i j}^{a}-\omega_{0}^{a} D_{i} e_{j}^{a}\right),
$$

where $D_{i}$ is a covariant derivative given by $\omega_{i}^{a}$ and $F_{i j}^{a}$ is its curvature. From this expression, one sees that $\left(\omega_{i}, \epsilon^{i j} e_{j}\right)$ are cannonically cojugate to each other, while $e_{0}$ and $\omega_{0}$ are Lagrange multipliers and impose constraints, $F_{i j}=0$ and $D_{i} e_{j}-D_{j} e_{i}=0$. Thus a wave-function of the theory can be represented by a function $\Phi(\omega)$ of $\omega_{i}$, a $S O(3)$ connection on $\Sigma$. The constraint $F_{i j}=0$ implies that $\Phi(\omega)$ should vanish unless $\omega$ is flat, and $\epsilon^{i j} D_{i} e_{j} \Phi(\omega)=i D_{i} \frac{\delta}{\delta \omega_{i}} \Phi(\omega)=0$ means that $\Phi(\omega)$ is invariant under the gaugetransformation $\omega_{i} \rightarrow \omega_{i}+D_{i} \lambda$. The inner product in $H_{C S}$ is given by the integral

$$
\left(\Phi_{1}, \Phi_{2}\right)_{C S}=\int[d \omega] \delta\left(F_{i j}\right) \Phi_{1}^{*}(\omega) \Phi_{2}(\omega)
$$

Thus a physical wave-function is a half-density on the moduli space of a flat $S O(3)$ connection.

Now we would like to show that there is a natural isomorphism between $H(\Delta)$ and $H_{C S}$. To interpolate between the two Hilbert spaces, we introduce the following (overcomplete) basis for $H_{C S}$ constructed from Wilson-lines $U_{j}(x, y)(x, y \in \Sigma, j=0,1,2, \ldots)$,

$$
U_{j}(x, y)=P \exp \left(\int_{x}^{y} \omega^{a} t_{j}^{a}\right),
$$

where $P \exp$ denotes the path-ordered exponential and $t_{j}^{a}(a=1,2,3)$ is the spin- $j$ generator of $S O(3)$. Under a gauge transformation $\omega \rightarrow \Omega^{-1} \omega \Omega+\Omega^{-1} d \Omega$, the Wilson-line behaves as $U(x, y) \rightarrow \Omega(x)^{-1} U(x, y) \Omega(y)$. Now consider their tensor product $\otimes_{i} U_{j_{i}}\left(x_{i}, y_{i}\right)$. 
To make this gauge-invariant, we need to contract group indices of $U_{j}$ 's so that the gauge factor $\Omega$ cancels out. In the case of the group $S O(3)$, invariant tensors we can use to contract group indices are the Clebsch-Gordan coefficient $\left\langle j_{1} j_{2} m_{1} m_{2} \mid j_{3} m_{3}\right\rangle$ and the metric

$$
\begin{aligned}
g_{m m^{\prime}}^{j} & =(-1)^{j-m} \frac{1}{\sqrt{2 j+1}} \delta_{m+m^{\prime}, 0} \\
g_{j}^{m m^{\prime}} & =(-1)^{j+m} \sqrt{2 j+1} \delta_{m+m^{\prime}, 0} .
\end{aligned}
$$

Actually it is more convenient to use the cyclic-symmetric $3 j$-symbol given by

$$
\left(\begin{array}{ccc}
j_{1} & j_{2} & j_{3} \\
m_{1} & m_{2} & m_{3}
\end{array}\right)=\frac{(-1)^{j_{1}-j_{2}-m_{3}}}{\sqrt{2 j_{3}+1}}\left\langle j_{1} j_{2} m_{1} m_{2} \mid j_{3}-m_{3}\right\rangle,
$$

rather than the Clebsch-Gordan coefficient. We regard $m_{i}$ 's in the $3 j$-symbol as lower indices which can be raised by the metric $g_{j_{i}}^{m_{i} m_{i}^{\prime}}$. When three Wilson-lines meet together at the same point on $\Sigma$, we can use the $3 j$-symbol and the metric $g_{m m^{\prime}}^{j}$ to contract their group indices. We can also connect two Wilson-lines by the metric if they carry the same spin. The gauge-invariant function constructed this way corresponds to a colored trivalent graph $Y$ on $\Sigma$, where a contour from $x$ to $y$ in $Y$ with color $j$ corresponds to a Wilson-line $U_{j}(x, y)$, and a three-point vertex in $Y$ represents the $3 j$-symbol ${ }^{\star}$. Due to the cyclic symmetry of the $3 j$-symbol, to each graph $Y$ on the orientable surface $\Sigma$, we can associate such a gauge-invariant function uniquely.

A physical wave-function of the Chern-Simons theory is obtained from such a network of Wilson-lines by restricting the support of the function on flat $S O(3)$ connections. This restriction however gives rise to linear dependence among the Wilson-line networks. Specifically, if two graphes $Y$ and $Y^{\prime}$ are homotopic, the corresponding gauge-invariant functions have the same value on a flat connection. Since there is one to one correspondence between a homotopy class of colored trivalent graphes on $\Sigma$ and a triangulation of $\Sigma$ with coloring on their sides, we may parametrize the gauge-invariant function by a colored triangulation

* There may be a pair of Wilson-lines intersecting with each other, which cannot be described as a part of a trivalent graph as it is. In such a case, we may cut the Wilson-lines at the intersecting point and use the orthonormality of the $3 j$-symbols,

$$
\delta_{m_{1}, m_{1}^{\prime}} \delta_{m_{2}, m_{2}^{\prime}}=\sum_{j_{3}, m_{3}}\left(2 j_{3}+1\right)\left(\begin{array}{ccc}
j_{1} & j_{2} & j_{3} \\
m_{1} & m_{2} & m_{3}
\end{array}\right)\left(\begin{array}{ccc}
j_{1} & j_{2} & j_{3} \\
m_{1}^{\prime} & m_{2}^{\prime} & m_{3}
\end{array}\right)
$$

to replace the intersection by two vertices and an infinitesimal Wilson-line connecting the vertices. 
defined by a pair $(\Delta, c)(c \in C(\Delta))$ rather than a trivalent graph $Y$. In this way, to each colored triangulation, we can associate a physical wave-function $\Psi_{\Delta, c}$ of the Chern-Simons theory. An arbitrary wave-function $\Phi(\omega)$ is expanded in terms of them as

$$
\Phi(\omega)=\sum_{\Delta} \sum_{c \in \Delta} \varphi_{\Delta}(c) \Lambda^{-n(\Delta)} \Psi_{\Delta, c}(\omega)
$$

Now we are in a position to establish a correspondence between a solution to the discretized Wheeler-DeWitt equation (10) and a physical state in the Chern-Simons theory. To understand the correspondence, the following fact is most important. When evaluated on a flat connection $\omega, \Psi_{\Delta, c}(\omega)$ are not yet linearly independent, but they obey the following relations,

$$
\Psi_{\Delta, c}(\omega)=\sum_{c^{\prime} \in C\left(\Delta^{\prime}\right)} P_{\Delta, \Delta^{\prime}}\left(c, c^{\prime}\right) \Lambda^{-n\left(\Delta^{\prime}\right)} \Psi_{\Delta^{\prime}, c^{\prime}}(\omega)
$$

Furthermore they are the only linear relations on $\Psi_{\Delta, c}$.

Before proving (15), let us examine its consequences. By substituting (15) into (14), we obtain

$$
\Phi(\omega)=\sum_{c \in C(\Delta)} \phi_{\Delta}(c) \Lambda^{-n(\Delta)} \Psi_{\Delta, c}(\omega)
$$

where $\phi_{\Delta}(c)$ is defined by

$$
\phi_{\Delta}(c)=\sum_{\Delta^{\prime}} \sum_{c^{\prime} \in C\left(\Delta^{\prime}\right)} P_{\Delta, \Delta^{\prime}}\left(c, c^{\prime}\right) \Lambda^{-n\left(\Delta^{\prime}\right)} \varphi_{\Delta^{\prime}}\left(c^{\prime}\right)
$$

for an arbitrary fixed triangulation $\Delta$ of $\Sigma$. It follows from $(9)$ that $\phi_{\Delta}(c)$ solves the Wheeler-DeWitt equation (10) of the lattice gravity.

$$
\phi_{\Delta}=\mathcal{P}\left[\phi_{\Delta}\right]
$$

Thus, to each solution $\phi_{\Delta}(c)$ of the Wheeler-DeWitt equation, there is a physical state $\Phi(\omega)$ of the Chern-Simons theory given by (16). Since (15) are the only relations among $\Psi_{\Delta, c}$ 's, this correspondence between $\phi_{\Delta}$ and $\Phi$ is one to one. In Section 4 , we will show 
that the inner product of Wilson-line networks $\left(\Psi_{\Delta_{1}, c_{1}}, \Psi_{\Delta_{2}, c_{2}}\right)_{C S}$ in the Chern-Simons theory is equal to $P_{\Delta_{1}, \Delta_{2}}\left(c_{1}, c_{2}\right)$ for the lattice gravity, upto a constant factor. Therefore the map from $H(\Delta)$ to $H_{C S}$ defined by (16) preserves their inner products. Thus (16) gives the isomorphism between the physical Hilbert spaces of the lattice gravity and the Chern-Simons theory.

Now we would like to prove that the relations (15) indeed hold, and that they are the only relations among $\Psi_{\Delta, c}$ 's. We will show this by mathematical induction with respect to the number of tetrahedra in $N=\Sigma \times[0,1]$. When the number is zero, the triangulations $\Delta$ and $\Delta^{\prime}$ must be identical and they are attached to each other. In this case, (15) is an obvious identity. Now we are going to pile tetrahedra one on another and increase the number of tetrahedra in $N$. Since one tetrahedra has four faces, there are three ways to attach one on another.

(i) Choose one of the faces of the tetrahedron and attach it to one of the triangles on the surface $\Sigma$ of $N$ (Fig. 5).

(ii) Attach two faces of the tetrahedron to two neighbouring triangles on $\Sigma$ (Fig. 6).

(iii) Attach three faces of the tetrahedron to three neighbouring triangles on $\Sigma$ (figure obtained by inverting the arrows in Fig. 5).

Let us first check that the induction holds in the second move in the above list. Consider a part of the Wilson-line network of $\Psi_{\Delta, c}$ which looks like the diagram in the right hand side of Fig. 6. Because of the flatness of $\omega$, we can take the Wilson-line colored by $k$ and make its length to be arbitrary small without changing the value of $\Psi_{\Delta, c}(\omega)$. When its end-points meet with each other, the Wilson-line can be replace by an identity. Since the group indices of the Wilson-line $U_{k}(x, y)$ at the end-points $x$ and $y$ are contracted with the $3 j$-symbols, in the limit $x \rightarrow y$ when $U_{j}(x, y)$ becomes an identity, the function $\Psi_{\Delta, c}$ should contain a sum of product of these $3 j$-symbols. Now there is a formula which relate two different ways of summing $3 j$-symbols, 


$$
\begin{gathered}
\sum_{m m^{\prime}} g_{k}^{m m^{\prime}}\left(\begin{array}{ccc}
j_{2} & j_{3} & k \\
m_{2} & m_{3} & m
\end{array}\right)\left(\begin{array}{ccc}
j_{4} & j_{1} & k \\
m_{4} & m_{1} & m^{\prime}
\end{array}\right) \\
=\sum_{l}(-1)^{j_{1}+j_{2}+j_{3}+j_{4}} \sqrt{(2 k+1)(2 l+1)}\left\{\begin{array}{ccc}
j_{1} & j_{2} & l \\
j_{3} & j_{4} & k
\end{array}\right\} \\
\times \sum_{n n^{\prime}} g_{l}^{n n^{\prime}}\left(\begin{array}{ccc}
j_{1} & j_{2} & l \\
m_{1} & m_{2} & n
\end{array}\right)\left(\begin{array}{ccc}
j_{3} & j_{4} & l \\
m_{3} & m_{4} & n^{\prime}
\end{array}\right)
\end{gathered}
$$

The left hand side of this equation corresponds to the diagram in the left hand side of Fig. 6. These four external Wilson-lines are recombined in the right hand side; the Wilson-lines of $j_{1}$ and $j_{2}$ make a pair and they are connected to $j_{3}$ and $j_{4}$ by an infinitesimal Wilson-line with color- $l$. This is exactly the right hand side of Fig. 6. Therefore we obtain

$$
\Psi_{\Delta, c_{k}}=\sum_{l}(-1)^{j_{1}+j_{2}+j_{3}+j_{4}} \sqrt{(2 k+1)(2 l+1)}\left\{\begin{array}{ccc}
j_{1} & j_{2} & l \\
j_{3} & j_{4} & k
\end{array}\right\} \Psi_{\widetilde{\Delta}, \widetilde{c_{l}}}
$$

where the triangulation $\left(\Delta, c_{k}\right)$ contains two triangles colored as in the left hand side of Fig. 6 , and it is replaced by the ones in the dual position in $\left(\widetilde{\Delta}, \widetilde{c}_{l}\right)$.

To prove (15) inductively, suppose that we have used $n$-tetrahedra in constructing the projection operator $P$ for the topology $N=\Sigma \times[0,1]$. When we add one more tetrahedron to $N$, as is prescribed in (ii), the corresponding projection operator $P^{\prime}$ is obtained from $P$ by multiplying to it an appropriate factor involving the $6 j$-symbol, and by summing over coloring on the common side of two neighbouring triangles to which the new tetrahedron is attached. This operator $P^{\prime}$ is obtained exactly by substituting (18) into the right hand side of (15). Therefore the inductive proof of (15) holds when we add one tetradedron in the second move in the list.

We can also add a tetrahedron as in (i) or (iii) in the list. If the network contains a contractable loop with several external Wilson-lines attached, by repeatedly using the identity (17), the loop can be recombined into a tree-like diagram with a one-loop tadpole. The tadpole can be made arbitrarily small, and the infinitesimal tadpole can be removed by using

$$
\sum_{m m^{\prime}} g_{j}^{m m^{\prime}}\left(\begin{array}{ccc}
j & j & J \\
m & m^{\prime} & M
\end{array}\right)=\delta_{J, 0} \delta_{M, 0}
$$

For example, if the network defined by $(\Delta, c)$ contains a loop with three external lines $j_{1}$, 
$j_{2}$ and $j_{3}$ as in the right hand side of Fig. 5, we man shrink the loop to obtain another network $\left(\Delta^{\prime}, c^{\prime}\right)$ where the three lines meet at one point. By using the formula,

$$
\begin{aligned}
\sum_{n_{i j}} g_{l_{12}}^{n_{12} n_{12}^{\prime}} g_{l_{23} n_{23} n_{23}^{\prime}}^{\prime} g_{l_{31}}^{n_{31} n_{31}^{\prime}}\left(\begin{array}{ccc}
l_{12} & j_{2} & l_{23} \\
n_{12}^{\prime} & m_{2} & n_{23}
\end{array}\right)\left(\begin{array}{ccc}
l_{23} & j_{3} & l_{31} \\
n_{23}^{\prime} & m_{3} & n_{31}
\end{array}\right)\left(\begin{array}{ccc}
l_{31} & j_{1} & l_{12} \\
n_{31}^{\prime} & m_{1} & n_{12}
\end{array}\right) \\
=(-1)^{j_{1}+j_{2}+j_{3}} \sqrt{\left(2 l_{12}+1\right)\left(2 l_{23}+1\right)\left(2 l_{31}+1\right)} \\
\times\left\{\begin{array}{ccc}
j_{1} & j_{2} & j_{3} \\
l_{23} & l_{31} & l_{12}
\end{array}\right\}\left(\begin{array}{ccc}
j_{1} & j_{2} & j_{3} \\
m_{1} & m_{2} & m_{3}
\end{array}\right),
\end{aligned}
$$

we can relate the corresponding functions $\Psi_{\Delta, c}$ and $\Psi_{\Delta^{\prime}, c^{\prime}}$ as

$$
\Psi_{\Delta, c}=(-1)^{j_{1}+j_{2}+j_{3}} \sqrt{\left(2 l_{12}+1\right)\left(2 l_{23}+1\right)\left(2 l_{31}+1\right)}\left\{\begin{array}{lll}
j_{1} & j_{2} & j_{3} \\
l_{23} & l_{31} & l_{12}
\end{array}\right\} \Psi_{\Delta^{\prime}, c^{\prime}},
$$

where $l_{i j}$ is the color of the segment of the loop in $(\Delta, c)$ connecting $j_{i}$ and $j_{j}$. This corresponds to (iii) in the list, and the inductive proof holds in this move. The induction for the move (i) is also guaranteed by the same equation (19).

In this way, we have proved that the identities (15) holds forn arbitrary pair of $\Delta$ and $\Delta^{\prime}$. Using a variation of the analysis in Appendix D of[11], one can show that all other relations among $\Psi_{\Delta, c}$ on a flat connection $\omega$ are generated from (18) and (19). Therefore (15) are the only relations among $\Psi_{\Delta, c}$ 's.

\section{The Hartle-Hawking-Type Wave-Function}

In the previous section, we defined the isomorphism between the physical Hilbert spaces of the lattice gravity and the Chern-Simons theory. In this section, we will show that this isomorphism indeed identifies wave-functions associated to the same geometry of the threedimensional manifold. The geometry we consider here is a handlebody $M$. To describe $M$, we embed a closed orientable two-dimensional surface $\Sigma$ into $\mathbf{R}^{3}$. The handlebody $M$ is taken as the interior of $\Sigma$. Associated to such a geometry, we can construct physical states in both the lattice gravity and the Chern-Simons theory.

In the Chern-Simons theory, the physical wave-function $\Phi_{M}$ for $M$ is defined as

$$
\Phi_{M}\left(\omega_{\mid \Sigma}\right) \delta\left(F_{i j \mid \Sigma}\right)=\int_{\omega_{\mid \Sigma}: \text { fixed }}[d e, d \omega] \exp \left(i \int_{M} e \wedge(d \omega+\omega \wedge \omega)\right)
$$

where we perform the functional integral over $e$ and $\omega$ in the interior of $M$ with a fixed 
boundary condition of $\omega$ on $\partial M=\Sigma$. The integration over $e_{\mid \Sigma}$ gives rise to $\delta\left(F_{i j \mid \Sigma}\right)$ which is explicitly written in the left hand side of (20). This ensures that the functional integral in the right hand side gives a physical state of the Chern-Simons theory. Such a wavefunction may be regarded as a generalization of the Hartle-Hawking wave-function (The original wave-function of Hartle and Hawking[12] corresponds to the case when $\Sigma$ is $S^{2}$ and the handlebody $M$ is a three-dimensional ball.). The wave-function for the lattice gravity is defined in a similar fashion by fixing a triangulation $\Delta$ and its coloring $c$ of $\Sigma$, and by summing over all possible coloring in the interior of $M$. This is nothing but $Z_{M, \Delta}(c)$ we have introduced in Section 2.

In the previous section, we have found that, to each physical state $\phi_{\Delta}(c)$ of the lattice gravity, there is a corresponding state in the Chern-Simons theory defined by (16). Therefore it is natural to expect that the wave-functions $\Phi_{M}\left(\omega_{\mid \Sigma}\right)$ and $Z_{\Delta, M}(c)$ associated to the same handlebody $M$ are related as

$$
\Phi_{M}\left(\omega_{\mid \Sigma}\right)=A_{g} \sum_{c \in C(\Delta)} Z_{M, \Delta}(c) \Lambda^{-n(\Delta)} \Psi_{\Delta, c}\left(\omega_{\mid \Sigma}\right)
$$

when $\omega_{\mid \Sigma}$ is a flat connection. Here $A_{g}$ is a constant depending only on the genus of the handlebody $M$. This indeed is the case as we shall see below.

As was shown by Witten in the case of the Lorentzian Einstein gravity[13], there is a fairly explicity expression for the Hartle-Hawking-type wave-function $\Phi_{M}\left(\omega_{\mid \Sigma}\right)$. As well as the constraint $F_{i j \mid \Sigma}=0$ written explicitly in (20), the integration over $e$ in (20) imposes that $\Phi_{M}$ should vanish unless $\omega_{\mid \Sigma}$ have a flat extension $\omega$ interior of the handlebody $M$. This condition can be rephrased as follows. If the boundary $\Sigma$ of $M$ is of genus $g$, it has $2 g$ homology cycles. Among these, there are $g$ cycles which are contractable in $M$ while other $g$ cycles are not. The necessary and sufficient condition for $\omega_{\mid \Sigma}$ to have a flat extension in $M$ is that its holonomies $U_{(a)}(a=1, \ldots, g)$ around these contractable cycles are trivial. Therefore

$$
\Phi_{M}\left(\omega_{\mid \Sigma}\right)=A_{g}^{\prime} \prod_{a=1}^{g} \delta\left(U_{(a)}-\mathbf{1}\right),
$$

where $A_{g}^{\prime}$ is a constant independent of $\omega_{\mid \Sigma}$, and $\delta(U-\mathbf{1})$ is a $\delta$-function with respect to the Haar measure of $S O(3)$. Thus in order to prove the identity (21), we need to show 
that the sum over coloring in the right hand side of the equation imposes the constraint $U_{(a)}=1$ on $\omega_{\mid \Sigma}$. In the following we will show that, by recombining the Wilson-lines, the sum in the right hand side of (21) reduces to sums over colorings of the contractable cycles as

$$
\sum_{c \in C(\Delta)} Z_{M, \Delta}(c) \Lambda^{-n(\Delta)} \Psi_{\Delta, c}\left(\omega_{\mid \Sigma}\right)=\prod_{a=1}^{g}\left[\sum_{j=0}^{\infty}(2 j+1) \operatorname{Tr}\left(U_{(a)} j\right)\right] .
$$

The orthonormality and the completeness of the irreducible $S O(3)$ characters[14] imply that the right hand side of this equation gives the product of the $\delta$-functions as in (22).

Now we would like to prove the equation (23). Suppose we have used $n$-tetrahedra in computing the wave-function $Z_{\Delta, M}(c)$ for the handlebody $M$. The tetrahedra must have been placed in such a way that the boundary $\Sigma$ of $M$ is triangulated as $(\Delta, c)$. Let us choose one of the tetrahedra attached on the boundary surface. Since $\omega_{\mid \Sigma}$ is flat, we can use (15) to remove this tetrahedron, i.e.

$$
\sum_{c \in C(\Delta)} Z_{M, \Delta}(c) \Lambda^{-n(\Delta)} \Psi_{\Delta, c}\left(\omega_{\mid \Sigma}\right)=\sum_{c^{\prime} \in C\left(\Delta^{\prime}\right)} Z_{M, \Delta^{\prime}}(c) \Lambda^{-n\left(\Delta^{\prime}\right)} \Psi_{\Delta^{\prime}, c^{\prime}}\left(\omega_{\mid \Sigma}\right),
$$

where $\Delta$ is the original triangulation of $\Sigma$ in (23), and $\Delta^{\prime}$ is the one which is obtained by removing the tetrahedron attached on $\Sigma$. In computing $Z_{M, \Delta^{\prime}}(c)$, the number of tetrahedra we use is $(n-1)$. By repeating this procedure, we can eliminate all the tetrahedra in $M$.

To visualize this process, it is useful to imagine the handlebody $M$ as a balloon whose surface is of genus $g$. For example, when $\Sigma$ is a torus, we consider a tube of a tire. Removing the tetrahedra is then like reducing the air from the balloon. After gradually decreasing its volume, the balloon will eventually be flattened. To describe the flattened balloon, we note that the surface $\Sigma$ can be constructed from two discs with $g$ holes, $S_{g}^{+}$ and $S_{g}^{-}$, by gluing their boundaries together as shown in Fig. 7. We call $S_{g}^{+}$and $S_{g}^{-}$ as upper and lower parts of $\Sigma$. The boundaries of the $g$ holes in $S_{g}^{ \pm}$correspond to the homology cycles on $\Sigma$ which are not contractable in $M$. In the limit when the balloon is flattened, the upper and the lower parts of $\Sigma$ overlap one on another. Reflecting the original tetrahedral decomposition of $M, S_{g}^{ \pm}$are covered by triangles. It is not difficult to see that the triangulations of $S_{g}^{+}$and $S_{g}^{-}$must be identical and that they must have the same coloring. Namely the Wilson-line network in the upper part of $\Sigma$ is the mirror image of the one in the lower part as shown in Fig. 8. 
Let us perform the sum over colorings of the Wilson-lines across the boundaries of $S_{g}^{+}$ and $S_{g}^{-}$(for example the Wilson-line $j_{3}$ in Fig. 8). As we did in the previous section, we may take the lengths of these Wilson-lines arbitrarily small and replace them by $\mathbf{1}$. Because of the reflection symmetry of the Wilson-lines, we may use the orthonormality of the $3 j$-symbols

$$
\begin{gathered}
\sum_{j_{3}, m_{3}, m_{3}^{\prime}}(-1)^{j_{3}} \sqrt{2 j_{3}+1} g_{j_{3}}^{m_{3} m_{3}^{\prime}}\left(\begin{array}{ccc}
j_{1} & j_{2} & j_{3} \\
m_{1} & m_{2} & m_{3}
\end{array}\right)\left(\begin{array}{ccc}
j_{3} & j_{2} & j_{1} \\
m_{3}^{\prime} & m_{2}^{\prime} & m_{1}^{\prime}
\end{array}\right) \\
=(-1)^{j_{1}+j_{2}} \sqrt{\left(2 j_{1}+1\right)\left(2 j_{2}+1\right)} g_{m_{1} m_{1}^{\prime}}^{j_{1}} g_{m_{2} m_{2}^{\prime}}^{j_{2}}
\end{gathered}
$$

to emilinate the trivalent vertices at the end-points of the Wilson-lines (as shown in Fig. 9). Repeating this procedure, we can remove the vertices on $\Sigma$ one by one.

To understand how the resulting Wilson-line network looks like, let us examine the case when $\Sigma$ is a torus, in detail. In this case, its upper and lower parts are topologically the same as annuli, and each of them can be decomposed into two triangles as shown in Fig. 10. Corresponding to this triangulations, there are six Wilson-lines on $\Sigma$ which are connected by four vertices (Fig. 11a). We can choose one of the Wilson-lines, say $j_{2}$ in Fig. 11a, and remove a pair of vertices at its end-points by using (24). As the result, we obtain a diagram as shown in Fig. 11b. Because of the flatness of $\omega_{\mid \Sigma}$, we can move around the Wilson-line $j_{1}$ homotopically, and the network in Fig. 11b can be brought into the one in Fig. 11c. Now the Wilson-loop consisting of $j_{1}$ and $j_{3}$ is contractable on $\Sigma$, and we end up in Fig. 11d. In this way, the Wilson-line network on the torus is deformed into a single Wilson-loop around its homology cycle contractable in $M$, as shown in Fig. 11e. Taking into account the weight $Z_{M, \Delta}(c)$, we have checked that the resulting summation over $j_{4}$ reproduces the right hand side of $(23)$ for $g=1$.

For $g \geq 2$, we can, for example, choose a triangulation of $S_{g}^{ \pm}$as in Fig. 12a. The corresponding Wilson-line network is shown in Fig. 12b. As in the case of the torus described in the above, one can follow the deformation of the network and show that $\sum_{c} Z_{M, \Delta}(c) \Psi_{\Delta, c}$ for this triangulation $\Delta$ reduces to the right hand side of (23). This proves the identity (21), and we found the factor $A_{g}$ is equal to $A_{g}^{\prime}$ which is related to the normalization of the path integral (20). 
We have found that the Hartle-Hawking-type wave-functions in the lattice gravity and the Chern-Simons theory are related by the isomorphism (14) between the physical Hilbert spaces of the two theories. In this section, we will exploit this result to show that, for any closed orientable manifold $M$, the partition functions of the two theories agree with each other. The idea is to use the Heegard splitting of $M[15]$. Consider two handlebodies $M_{1}$ and $M_{2}$ whose boundaries are of the same topology $\Sigma$. Since $M_{1}$ and $M_{2}$ differ only by the markings of the homology cycles on their boundaries, we can glue the boundaries together by their diffeomorphism and obtain a closed three-dimensional manifold. Moreover it is known that any closed manifold can be realized in this way. In this construction, the topology of $M$ is encoded into the topology of $\partial M_{1}$ and $\partial M_{2}$ and how they are glued together.

Corresponding to this splitting of $M$, the partition function of the Chern-Simons theory is expressed as an inner product of the Hartle-Hawking-type wave functions $\Phi_{M_{1}}$ and $\Phi_{M_{2}}$,

$$
Z_{M}^{(C S)}=\left(\Phi_{M_{1}}, \Phi_{M_{2}}\right)_{C S}
$$

as far as $M$ is orientable. This formula is derived from the functional integral expression for $Z_{M}^{(C S)}$; the functional integrals over $M_{1}$ and $M_{2}$ result in the Hartle-Hawking-type wave-functions $\Phi_{M_{1}}$ and $\Phi_{M_{2}}$, and the functional integral on the boundary $\partial M_{1} \simeq \partial M_{2}$ corresponds to taking their inner product. On the other hand, the partition function for the lattice gravity has also the expression

$$
Z_{M}=\left(Z_{M_{1}, \Delta}, Z_{M_{2}, \Delta}\right)
$$

as we saw in Section 2. Since the Hartle-Hawking-type wave-functions in the Chern-Simons theory and the lattice gravity are related by $(21), Z_{M}^{(C S)}$ and $Z_{M}$ are the same provided the isomorphism (14) preserves the inner products in the two Hilbert spaces, $H_{C S}$ and $H(\Delta)$. Thus, in order to establish the equivalence $Z_{M}^{(C S)}=Z_{M}$, we want to show

$$
\left(\Psi_{\Delta_{1}, c_{1}}, \Psi_{\Delta_{2}, c_{2}}\right)_{C S}=A_{g}^{2} \cdot P_{\Delta_{1}, \Delta_{2}}\left(c_{1}, c_{2}\right)
$$


or equivalently

$$
\begin{gathered}
\sum_{\substack{c_{1} \in C\left(\Delta_{1}\right) \\
c_{2} \in C\left(\Delta_{2}\right)}} \Psi_{\Delta_{1}, c_{1}}\left(\omega_{1}\right) \Lambda^{-n\left(\Delta_{1}\right)} P_{\Delta_{1}, \Delta_{2}}\left(c_{1}, c_{2}\right) \Lambda^{-n\left(\Delta_{2}\right)} \Psi_{\Delta_{2}, c_{2}}\left(\omega_{2}\right) \\
=A_{g}^{-2} \cdot K\left(\omega_{1}, \omega_{2}\right)
\end{gathered}
$$

where $K\left(\omega_{1}, \omega_{2}\right)$ is a kernel for the inner product

$$
\left(\Phi, \Phi^{\prime}\right)_{C S}=\int\left[d \omega_{1}\right] \delta\left(F_{1, i j}\right) \int\left[d \omega_{2}\right] \delta\left(F_{2, i j}\right) \Phi\left(\omega_{1}\right) K\left(\omega_{1}, \omega_{2}\right) \Phi\left(\omega_{2}\right)
$$

and it is given in term of the functional integral

$$
K\left(\omega_{1}, \omega_{2}\right) \delta\left(F_{1, i j}\right) \delta\left(F_{2, i j}\right)=\int_{\substack{\omega(t=0)=\omega_{1} \\ \omega(t=1)=\omega_{2}}}[d e, d \omega] \exp \left(i S_{C S}(e, \omega)\right)
$$

for the topology $N=\Sigma \times[0,1]$.

Now we are going to show that the left hand side of (28) is proportional to the right hand side. The factor $A_{g}^{-2}$ will be fixed later. Since $\Psi_{\Delta_{i}, c_{i}}$ is evaluated on a flat connection $\omega_{i}$, we may use (15) to rewrite the left hand side of (28) as

$$
\sum_{c \in C(\Delta)} \Lambda^{-n(\Delta)} \Psi_{\Delta, c}\left(\omega_{1}\right) \Psi_{\Delta, c}\left(\omega_{2}\right)
$$

On the other hand, it follows from the functional integral expression (29) that the kernel $K\left(\omega_{1}, \omega_{2}\right)$ vanishes unless $\omega_{1}$ and $\omega_{2}$ has a flat extension in $N$. For $N=\Sigma \times[0,1]$, the flat extension exists if and only if $\omega_{1}$ and $\omega_{2}$ are gauge-equivalent. Thus we need to show that the sum over coloring in (30) imposes the the constraint, $\omega_{1} \simeq \omega_{2}$

Let us study the case when $\Sigma$ is a torus, in detail. In this case, the surface $\Sigma$ can be decomposed into two triangles as shown in Fig. 13a. The corresponding network of Wilson-lines is shown in Fig. 13b. A flat connection $\omega$ on the torus can be specified by holonomies $U$ and $V$ around the two homology cycles on $\Sigma$. The wave-function $\Psi_{\Delta, c}$ for the network can then be regarded as a function of $U$ and $V$. In the network in Fig. 13b, 
the Wilson-line $j_{3}$ can be made arbitrarily short using the flatness of $\omega$ and be replaced by an identity. In this case, the wave-function $\Psi_{\Delta, c}$ is expressed as a function of $U$ and $V$ as

$$
\begin{aligned}
\Psi_{\Delta ; c}(U, V)= & \sum_{m_{i}, m_{i}^{\prime}, m_{i}^{\prime \prime}} U_{j_{1} m_{1}^{\prime}}^{m_{1}} V_{j_{2} m_{2}^{\prime}}^{m_{2}} \\
& \times g_{j_{1}}^{m_{1}^{\prime} m_{1}^{\prime \prime}} g_{j_{2}}^{m_{2}^{\prime} m_{2}^{\prime \prime}} g_{j_{3}}^{m_{3} m_{3}^{\prime \prime}}\left(\begin{array}{ccc}
j_{1} & j_{2} & j_{3} \\
m_{1} & m_{2} & m_{3}
\end{array}\right)\left(\begin{array}{ccc}
j_{1} & j_{2} & j_{3} \\
m_{1}^{\prime \prime} & m_{2}^{\prime \prime} & m_{3}^{\prime \prime}
\end{array}\right) .
\end{aligned}
$$

Here we marked the homology cycles on $\Sigma$ in such a way that the Wilson-lines $j_{1}$ and $j_{2}$ wind around cycles corresponding to the holonomies $U$ and $V$. The holonomies $U$ and $V$ commute with each other, so they can be diagonalized simultaneously. Since the wavefunction $\Psi_{\Delta, c}$ is invariant under the simultaneous conjugation, $U \rightarrow \Omega^{-1} U \Omega, V \rightarrow \Omega^{-1} V \Omega$, we can substitute diagonal matrices $U_{j m^{\prime}}^{m}=e^{i m \theta} \delta_{m^{\prime}}^{m}$ and $V_{j m^{\prime}}^{m}=e^{i m \varphi} \delta_{m^{\prime}}^{m}$ into $U$ and $V$ in (31).

Now we would like to perform the summation,

$$
\sum_{j_{1}, j_{2}, j_{3}} \Lambda^{-1} \Psi_{\Delta, c}\left(\theta_{1}, \varphi_{1}\right) \Psi_{\Delta, c}\left(\theta_{2}, \varphi_{2}\right),
$$

where $\left(\theta_{1}, \varphi_{1}\right)$ and $\left(\theta_{2}, \varphi_{2}\right)$ are phases of the holonomies $\left(U_{1}, V_{1}\right)$ and $\left(U_{2}, V_{2}\right)$ for $\omega_{1}$ and $\omega_{2}$. Although it is possible to do the summation for generic values of the phases, it is more instructive to study the cases when two among the four phases vanish. Actually it is enough to study these cases as we shall see below.

Let us consider the case when $V_{1}=V_{2}=1$. In this case, the wave-function $\Psi_{\Delta, c}\left(U_{i}, V_{i}\right)$ is simplified as

$$
\Psi_{\Delta, c}\left(U_{i}, V_{i}=\mathbf{1}\right)=\sqrt{\frac{\left(2 j_{1}+1\right)\left(2 j_{2}+1\right)\left(2 j_{3}+1\right)}{2 j_{1}+1}} \operatorname{Tr}\left(U_{i j_{1}}\right) .
$$

The summation (32) is then performed as

$$
\begin{aligned}
& \sum_{j_{1}, j_{2}, j_{3}} \Psi_{\Delta, c}\left(U_{1}, V_{1}=\mathbf{1}\right) \Psi_{\Delta, c}\left(U_{2}, V_{2}=\mathbf{1}\right) \\
& =\sum_{j_{1}} \operatorname{Tr}\left(U_{1 j_{1}}\right) \operatorname{Tr}\left(U_{2 j_{1}}\right) \cdot \Lambda^{-1} \cdot \frac{1}{2 j_{1}+1} \sum_{\left|j_{2}-j_{3}\right| \leq j_{1} \leq j_{2}+j_{3}}\left(2 j_{2}+1\right)\left(2 j_{3}+1\right) \\
& =\sum_{j_{1}} \operatorname{Tr}\left(U_{1 j_{1}}\right) \operatorname{Tr}\left(U_{2 j_{1}}\right)=\delta\left(U_{1}-U_{2}\right) .
\end{aligned}
$$

Here we have used the definition (5) of $\Lambda$ and the orthonormality of the irreducible char- 
acters $\operatorname{Tr}\left(U_{j_{1}}\right)$. Thus the sum over the coloring in the left hand side of (28) indeed imposes the constraint $U_{1}=U_{2}$ when $V_{1}=V_{2}$. It is straightforward to do the computations in other cases when $U_{2}=V_{2}=\mathbf{1}$ or $V_{1}=U_{2}=\mathbf{1}$, and we have found that the sum in (32) imposes $U_{1}=U_{2}$ and $V_{1}=V_{2}$ in both of these cases.

We have seen that the left hand side of (28) is proportional to $K\left(\omega_{1}, \omega_{2}\right)$ as far as two among the four phases are equal to zero. Let us relax this condition and suppose that they are not necessarily zero, but their ratios $\theta_{i} / \varphi_{i}(i=1,2)$ are rational numbers. Since (32) is invariant under the modular transformation of $\Sigma$, we can change the basis of the homology cycles in such a way that two among the four phases around the cycles become equal to zero. The summation in (32) then reduces to the computation in the above and we see that the constraints $\omega_{1} \simeq \omega_{2}$ arises upon the summation. In general, when the ratios are not necessarily rational, we can find a series of rational numbers which converges to $\theta_{i} / \varphi_{i}$. At each step in the series, the sum over the coloring in (32) gives the constraints $\omega_{1} \simeq \omega_{2}$. Thus it should also be the case in the limit of the series.

This result is extended to surfaces of higher genera as follows. A genus- $g$ surface $\Sigma$ can be constructed from a $4 g$-sided polygon by gluing its sides together as is indicated in Fig. 14. Correspondingly the surface is decomposed into $4 g$ triangles. As in the case of the torus, we can parametrize the flat connection $\omega$ on $\Sigma$ by its holonomies $U_{(a)}, V_{(a)}(a=1, \ldots, g)$ around the homology cycles $\alpha_{a}$ and $\beta_{a}$ as marked in Fig. 14. These holonomies are subject to the constraint,

$$
U_{(1)} V_{(1)} U_{(1)}^{-1} V_{(1)}^{-1} \cdots U_{(g)} V_{(g)} U_{(g)}^{-1} V_{(g)}^{-1}=\mathbf{1}
$$

In this case, the wave-function $\Psi_{\Delta, c}(\omega)$ is a product of $U_{(a)}$ 's and $V_{(a)}$ 's connected by the $3 j$-symbols. Especially it depends on $U_{(1)}$ as

$$
\begin{aligned}
\Psi_{\Delta, c}(\omega)= & \sum_{m_{i}, m_{i}^{\prime}, m_{i}^{\prime \prime}} U_{(1) j_{1} m_{1}^{\prime}}^{m_{1}} W^{m_{3} m_{4}} \\
& \times g_{j_{1}}^{m_{1}^{\prime} m_{1}^{\prime \prime}} g_{j_{2}}^{m_{2}^{\prime} m_{2}^{\prime \prime}}\left(\begin{array}{ccc}
j_{1} & j_{2} & j_{3} \\
m_{1} & m_{2} & m_{3}
\end{array}\right)\left(\begin{array}{ccc}
j_{1} & j_{2} & j_{4} \\
m_{1}^{\prime \prime} & m_{2}^{\prime} & m_{4}
\end{array}\right),
\end{aligned}
$$

where $W^{m_{3} m_{4}}$ is independent of $U_{(1)}$. The sum of $\Psi_{\Delta, c}\left(\omega_{1}\right) \Psi_{\Delta, c}\left(\omega_{2}\right)$ over $j_{1}$ and $j_{2}$ then imposes the constraint $U_{(1) 1}=U_{(1) 2}$ as in the case of the torus. The rest of the summation can be done inductively, and we obtain the constraint $\omega_{1} \simeq \omega_{2}$. 
We have found that the left hand side of $(28)$ is equal to $K\left(\omega_{1}, \omega_{2}\right)$ upto a constant factor $B_{g}$

$$
\begin{gathered}
\sum_{\substack{c_{1} \in C\left(\Delta_{1}\right) \\
c_{2} \in C\left(\Delta_{2}\right)}} \Psi_{\Delta_{1}, c_{1}}\left(\omega_{1}\right) \Lambda^{-n\left(\Delta_{1}\right)} P_{\Delta_{1}, \Delta_{2}}\left(c_{1}, c_{2}\right) \Lambda^{-n\left(\Delta_{2}\right)} \Psi_{\Delta_{2}, c_{2}}\left(\omega_{2}\right) \\
=B_{g} \cdot K\left(\omega_{1}, \omega_{2}\right) .
\end{gathered}
$$

Equivalently

$$
\left(\Psi_{\Delta_{1}, c_{1}}, \Psi_{\Delta_{2}, c_{2}}\right)_{C S}=B_{g}^{-1} \cdot P_{\Delta_{1}, \Delta_{2}}\left(c_{1}, c_{2}\right)
$$

By combining this with (21) and by using the expressions (25) and (26), we obtain

$$
Z_{M}^{(C S)}=A_{g}^{2} B_{g}^{-1} Z_{M}
$$

Now we would like to show that $B_{g}$ is equal to $A_{g}^{2}$. Since $A_{g}$ comes from the normalization of the functional integral (20), we must specify it in order to relate $B_{g}$ to $A_{g}$. Here we define the normalization in such a way that the Chern-Simons partition function for $S^{3}$ is equal to 1 . To show $A_{g}^{2} B_{g}^{-1}=1$, we note that $S^{3}$ can be constructed from two handlebodies of any genus $g$. Let us take a closed surface $\Sigma$ of genus $g$ and embed it into $S^{3}$. It is easy to see that both the interior and the exterior of $\Sigma$ are handlebodies of genus $g$. In this setting, the left hand side of (34) is equal to 1 due to the normalization convention of $Z_{M}^{(C S)}$. On the other hand, it follows from the definition of the lattice gravity that $Z_{M}$ for $M=S^{3}$ is also 1. In this way, we have shown $A_{g}^{2} B_{g}^{-1}=1$ for any value $g$. This proves the equality of the partition functions of the lattice gravity and the Chern-Simons theory.

Actually, there is a cavear here. In the above, we have assumed that the integral (25) and the sum (26) are convergent. This is not always the case. For example, when $M$ is of the topology $\Sigma \times S^{1}$, the partition function $Z_{M}$ of the lattice gravity is given by a trace over physical states

$$
\sum_{c \in C(\Delta)} \Lambda^{-n(\Delta)} P_{\Delta, \Delta}(c, c),
$$

where $\Delta$ is a triangulation of $\Sigma$. Namely the partition function $Z_{M}$ counts the number of physical states for $\Sigma$ which is infinite if $g \geq 1$. In this case, the partition function $Z_{M}^{(C S)}$ for the Chern-Simons theory also diverges as was pointed out by Witten [13]. There he has shown that the divergence occurs when $e \rightarrow \infty$, namely when the size of $M$ is large, and thus it is infrared in nature. 
So far, we have considered the case of a closed three-dimensional manifold $M$. It is also possible to consider a manifold with boundaries and discuss transition amplitudes between initial and final states.

We have already studied some of such processes in this paper. For example, $P_{\Delta_{1}, \Delta_{2}}\left(c_{1}, c_{2}\right)$ corresponds to the geometry $N \simeq \Sigma \times[0,1]$ for a transition of $\Sigma$ into $\Sigma$. The HartleHawking-type wave-function $Z_{\Delta, M}(c)$ can also be viewed as describing a transition of a point into a closed surface $\Sigma$ (or a creation of $\Sigma$ from nothing). In both of these cases, we have found that the amplitudes of the lattice gravity and the Chern-Simons theory are related by the isomorphis defined in Section 2 .

We can extend this analysis and study more elaborated transition processes involving a topology-change of $\Sigma$. In [13], Witten has examined the following situation in the case of the Chern-Simons theory. Consider $\Sigma_{\text {initial }}$ consisting of two components $\Sigma_{1}$ and $\Sigma_{2}$ of respective genus $g_{1}$ and $g_{2}$. One can construct a manifold $M$ which interpolates $\Sigma_{\text {initial }}$ to another surface $\Sigma_{\text {final }}$ of genus $g=g_{1}+g_{2}$ consisting of a single component, as follows. We first embed $\Sigma_{\text {final }}$ into $\mathbf{R}^{3}$ to obtain a handlebody $M_{0}$ of genus $g$. We then remove from $M_{0}$ handlebodies of genus $g_{1}$ and $g_{2}$ whose boundaries are $\Sigma_{1}$ and $\Sigma_{2}$. The remaining protion of $M_{0}$ gives the manifold $M$ whose boundaries are $\Sigma_{1}, \Sigma_{2}$ and $\Sigma_{\text {final }}$. A wavefunction for the initial surface $\Sigma_{\text {initial }}$ is spanned by products of functions $\Phi_{\Sigma_{1}}$ and $\Phi_{\Sigma_{2}}$ of flat connections on $\Sigma_{1}$ and $\Sigma_{2}$. The transition amplitude for $M$ then relates $\Phi_{\Sigma_{1}} \Phi_{\Sigma_{2}}$ to $\Phi_{\Sigma_{\text {final }}}$ for the final surface. It is shown in [13] that the relation is as follows.

$$
\Phi_{\Sigma_{\text {final }}} \sim \delta(A-\mathbf{1}) \Phi_{\Sigma_{1}} \Phi_{\Sigma_{2}}
$$

Here $A$ is an element of $S O(3)$ given in terms of holonomies $U_{(a)}$ and $V_{(a)}(a=1, . ., g)$ on $\Sigma_{\text {final }}$ as

$$
A=U_{(1)} V_{(1)} U_{(1)}^{-1} V_{(1)}^{-1} \cdots U_{\left(g_{1}\right)} V_{\left(g_{1}\right)} U_{\left(g_{1}\right)}^{-1} V_{\left(g_{1}\right)}^{-1},
$$

and the holonomies $U_{(1)}, \ldots, U_{\left(g_{1}\right)}$ and $V_{(1)}, \ldots, V_{\left(g_{1}\right)}$ correspond to the homology cycles on $\Sigma_{\text {final }}$ which are homotopically equivalent to the cycles on $\Sigma_{1}$ through the manifold $M$.

For the lattice gravity, the transition amplitude $Z_{\Delta_{1}, \Delta_{2} ; \Delta_{\text {final }}}\left(c_{1}, c_{2} ; c_{\text {final }}\right)$ for $M$ is given by summing over coloring of tetrahedra interior of $M$ while keeping fixed the colorings $c_{1}, c_{2}$ and $c_{\text {final }}$ on $\Sigma_{1}, \Sigma_{2}$ and $\Sigma_{\text {final }}$. To see its relation to the transition amplitude (35) 
in the Chern-Simons theory, we multiply the Wilson-line networks to $Z_{\Delta_{1}, \Delta_{2} ; \Delta_{\text {final }}}$ and sum over the colorings as

$$
\begin{aligned}
& \sum_{\substack{c_{1} \in C\left(\Delta_{1}\right), c_{2} \in C\left(\Delta_{2}\right) \\
C_{\text {final }} \in C\left(\Delta_{\text {final }}\right)}} Z_{\Delta_{1}, \Delta_{2} ; \Delta_{\text {final }}}\left(c_{1}, c_{2} ; c_{\text {final }}\right) \\
& \quad \times \Lambda^{-n\left(\Delta_{1}\right)} \Psi_{\Delta_{1}, c_{1}} \Lambda^{-n\left(\Delta_{2}\right)} \Psi_{\Delta_{2}, c_{2}} \Lambda^{-n\left(\Delta_{\text {final }}\right)} \Psi_{\Delta_{\text {fianl }}, c_{\text {final }}} .
\end{aligned}
$$

The computation is essentiall the same as we did in (28), and the result agrees with (35). Thus the transition ampltudes of this type are also equivalent in the two theories.

\section{Discussions}

We have found that the partition functions and some topology-changing amplitudes of the lattice gravity of Ponzano and Reggeare equal to the ones of the $I S O(3)$ ChernSimons theory. This result supports the original conjecture by Ponzano and Regge that the statistical sum of the $6 j$-symbols describes the fluctuating geometry with the weight $\prod_{x \in M} \cos (\sqrt{g} R)$. Indeed, if we integrate over $\omega$ first in the Chern-Simons functional integral, we are left with an integral over the dreibein $e$ with a weight $\exp \left(\int e \wedge R\right)$ where $R$ is a curvature two-form constructed from $e$. Although we seem to have gotten exp rather than cos, we should note that $\int e \wedge R$ changes its sign if we flip the orientation of $e$. Since we integrate over $e$ as a part of the $I S O(3)$ gauge field, at each point in $M$, both orientations of $e$ contribute to the functional integral. If one tries to integrate over $e$ of a fixed orientation, one would need to replace the exponential by the cosine to compensate for the restriction. Therefore it appears that the lattice gravity of Ponzano and Regge gives a functional integral of $\prod_{x \in M} \cos (\sqrt{g} R)$ with the correct measure for the fluctuating metric on $M$.

To regard this system as the Euclidean Einstein gravity, the factor $i$ in front of the action is disturbing. One cannot eliminate it by rotating the contour of the $e$-integral since the resulting functional integral would be divergent. To address this issue, it would be more fruitful to study a Lorentzian version of the lattice model based on the infinite dimensional representations of $S O(2,1)$. Since the representation theory of $S O(2,1)$ is far richer than that of $S O(3)$, we must have good criteria in choosing a class of representations we put of the edges of the tetrahedron. One of the criteria would be that a sum of characters over 
such representations should give the invariant $\delta$-function $\delta(U-\mathbf{1})$, in order for the lattice model to have the same partition function as in the Lorentzian Chern-Simons gravity. Such study will be useful in understanding the structure of physical observables in the Lorentzian gravity.

Recently Mizoguchi and Tada[16] have studied the $q$-analogue of the $6 j$-symbol and found the asymptotic formula for $q=e^{2 \pi i /(k+2)}$

$$
(-1)^{\sum_{i} j_{i}}\left\{\begin{array}{ccc}
j_{1} & j_{2} & j_{3} \\
j_{4} & j_{5} & j_{6}
\end{array}\right\}_{q} \sim \frac{c}{\sqrt{V}} \cos \left(S_{\text {Regge }}-\frac{\lambda_{k}}{2} V+\pi . / 4\right)
$$

where $c$ is some constant and $\lambda_{k}=(4 \pi / k)^{2}$. Thus it is natural to expect that the $q$-analogue of the Ponzano and Regge model introduced by Turaev and Viro would be related to the gravity with the cosmological constant $\lambda_{k}$ or the $S O(3) \times S O(3)$ Chern-Simons theory. The recent paper by Turaev [9] supports the latter possibility.

Durhuus, Jakobsen and Ryszard[17] have constructed a large class of topological lattice models extending the model of Turaev and Viro. The method developed in this paper could also be applicable to study those models.

\section{Acknowledgments}

The author would like to thank N. Sakakura for discussions. He is also thankful to T. Maskawa for explaining him some group theoretical facts and to L. Kauffman for encouragements.

\section{REFERENCES}

1. G. Ponzano and T. Regge, in Spectroscopic and Group Theoretical Methods in Physics, ed. F. Block (North-Holland, Amsterdam, 1968).

2. T. Regge, Nuovo Cimento 19 (1961) 558.

3. V.A. Kazakov, Mod. Phys. Lett. A4 (1989) 2125;

M.R. Douglas and S.H. Shenker, Nucle. Phys. B335 (1990) 635;

D.J. Gross and A.A. Migdal, Phys. Rev. Lett. 64 (1990) 127;

E. Brezin and V.A. Kazakov, Phys. Lett. 236B (1990) 144.

4. B. Hasslacher and M.J. Perry, Phys. Lett. 103B (1981) 21. 
5. V.G. Turaev and O.Y. Viro, "State Sum Invariants of 3-Manifolds and Quantum 6j-Symbols," preprint (1990).

6. E. Witten, Commun. Math. Phys. 121 (1989) 351.

7. H. Ooguri and N. Sasakura, Mod. Phys. Lett A6 (1991) 3591.

8. E. Witten, Nucl. Phys. B311 (1988/898) 46.

9. V.G. Turaev, "Quantum Invariants of 3-Manifolds and A Glimpse of Shadow Topology," hand-written manuscript (1991).

10. B.S. DeWitt, Phys. Rev. 160 (1967) 1113;

J.A. Wheeler, in Batelle Recontres, eds C.M. DeWitt and J.A. Wheeler (W.A. Benjamin, 1968).

11. B.V. Boulatov, V.A. Kazakov, I.K. Kostov and A.A. Migdal, Nucl. Phys. B273 [FS17] (1986) 641.

12. J. Hartle and S. Hawking, Phys. Rev. D28 (1983) 2960.

13. E. Witten, Nucl. Phys. B323 (1989) 113.

14. See for example, L.S. Pontryagin, Chapter 5 of "Topological Groups," the second edition, translated by A. Brown (Gordon and Breach, 1966).

15. J. Singer, Transactions of the American Math. Soc. 35 (1933) 88

16. S. Mizoguchi and T. Tada, Kyoto preprint, YITP/U-91-43 (1991).

17. B. Durhuus, H.P. Jakobsen and R. Nest, "Topological Quantum Field Theories from Generalized 6j-Symbols,", preprint (1991). 


\section{FIGURE CAPTIONS}

1. A tetrahedron colored by angular momenta $j_{i}$ 's.

2. One tetrahedron can be decomposed into four small tetradedra.

3. A manifold $M$ is decomposed into three parts, $M_{1}, M_{2}$ and $N$.

4. $Z_{M_{i}, \Delta_{i}}\left(c_{i}\right)$ is defined by the summation over colorings on edges interior of $M_{i}$ with the fixed coloring $c_{i}$ on the boundary.

5. Attaching a tetrahedron to a triangle on a surface, as seen from the above, and the corresponding Wilson-line networks.

6. Attaching a tetrahedron to two neighbouring triangles on a surface, as seen from the above, and the corresponding Wilson-line networks.

7. A genus- $g$ surface can be constructed from two discs with $g$-holes by gluing their boundaries together. The case of $g=3$ is shown in the figure.

8. Part of Wilson-lines on $S_{g}^{+}$and $S_{g}^{-}$.

9. The Wilson-line $j_{3}$ in Fig. 8 can be removed using the orthogonality of the $3 j$-symbols.

10. Triangulation of $S_{g=1}^{ \pm}$in the case of a torus.

11a. Wilson-line network corresponding to the triangulation in Fig. 10.

11b. The Wilson-line $j_{2}$ is removed using the orthogonality of the $3 j$-symbols.

11c. The Wilson-line $j_{1}$ can be moved around using the flatness of $\omega_{\mid \Sigma}$.

11d. The homotopically trivial loop is removed.

11e. The network is transformed into a single Wilson-loop around the homology cycle contractable in $M$. The sum over $j_{4}$ restricts the holonomy around this cycle to be trivial. 12a. Triangulation of $S_{g}^{ \pm}$.

12b. The corresponding Wilson-line network on $S_{g}^{ \pm}$.

13a. Torus can be decomposed into two triangles.

13b. The corresponding Wilson-line network.

14. A genus- $g$ surface can be constructed from a $4 g$-sided polygon. Correspondingly, the surface is decomposed into $4 g$ triangles. 